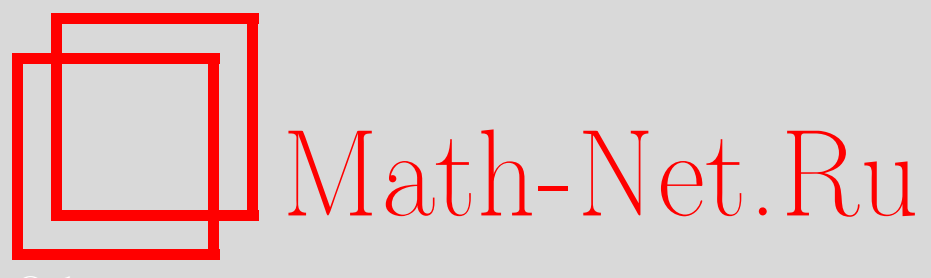

Ю. А. Алхутов, И. В. Асташова, В. И. Богачев, В. Н. Денисов, В. В. Козлов, С. Е. Пастухова, А. Л. Пятницкий, В. А. Садовничий, А. М. Степин, А. С. Шамаев, А. А. Шкаликов, Василий Васильевич Жиков (некролог), УМН, 2018, том 73, выпуск 3, 169-176

DOI: https://doi.org/10.4213/rm9826

Использование Общероссийского математического портала Math-Net.Ru подразумевает, что вы прочитали и согласны с пользовательским соглашением http://www . mathnet.ru/rus/agreement

Параметры загрузки:

IP: 54.80 .97 .219

26 апреля 2023 г., 15:40:08

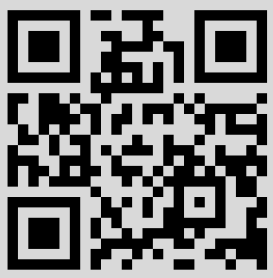




\section{Василий Васильевич Жиков}

12 февраля 2017 г. ушел из жизни выдающийся российский математик, профессор кафедры дифференциальных уравнений механико-математического факультета Московского государственного университета им. М.В. Ломоносова, профессор Владимирского государственного университета Василий Васильевич Жиков.

Василий Васильевич Жиков родился 14 августа 1941 г. в Новочеркасске. В 1958 г. он поступил на механико-математический факультет МГУ, который окончил в 1963 г. Затем он продолжил обучение в аспирантуре, завершившееся успешной защитой кандидатской диссертации в 1966 г. В том же году началась его более чем полувековая педагогическая работа: во Владимирском политехническом институте (старший преподаватель, доцент), затем во Владимирском педагогическом институте (позже Владимирском государственном гуманитарном университете, который в 2011 г. стал структурным

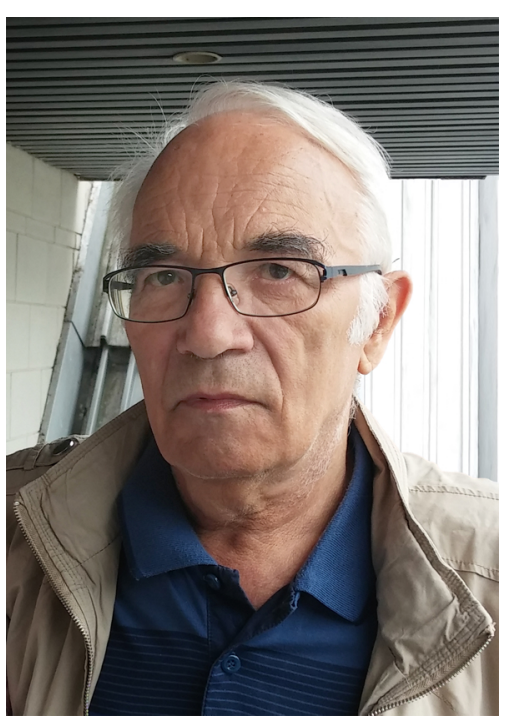
подразделением Владимирского государственного университета им. А. Г. и Н. Г. Столетовых), где он стал профессором в 1978 г. Докторскую диссертацию Василий Васильевич защитил в 1975 г. С 2000 г. он по совместительству работал также профессором кафедры дифференциальных уравнений механико-математического факультета МГУ, где участвовал в руководстве известным научным семинаром.

В. В. Жиков возглавлял Специализированный диссертационный совет при Владимирском государственном университете и заведовал кафедрой математического анализа и математической лабораторией. Под его руководством при лаборатории был организован научно-исследовательский семинар, который ныне известен далеко за пределами Владимира. Много сил отдал Василий Васильевич работе в экспертном совете ВАК. Многие математики следующих поколений с благодарностью вспоминают его как компетентного и требовательного, но в то же время благожелательного оппонента.

Работы В. В. Жикова в области дифференциальных уравнений, функционального анализа и математической физики оставили в математике глубокий след, некоторые из них, безусловно, стали классическими. Василий Васильевич справедливо признан ученым мирового уровня и является одним из самых высокоцитируемых российских математиков.

В. В. Жиков является автором более 200 научных работ (в базе MathSciNet записаны 183 его публикации), в том числе 4 монографии и 5 больших обзоров в журнале "Успехи математических наук".

DOI: https://doi.org/10.4213/rm9826 
Среди других наиболее известных и часто цитируемых его работ 15 статей в "Математическом сборнике", 9 статей в журнале "Известия РАН" и столько же в журнале "Функциональный анализ и его приложения" (список основных работ В. В. ЖКикова имеется в базе MathSciNet, частично на портале Math-Net.Ru и на сайте http://istina.msu.ru/profile/vvzhikov).

Научные интересы В.В. Жикова были весьма многосторонними, в разных областях он сумел добиться первоклассных достижений. Здесь отметим некоторые из них.

1. Основным направлением исследований Василия Васильевича в первое десятилетие его научной деятельности стали теория почти периодических функций и почти периодических решений уравнений в банаховом пространстве, а также применение методов функционального анализа и топологической динамики к операторным дифференциальным уравнениям. Соответствующий раздел теории почти периодических функций часто называют теорией Америо-Прузе-Жикова. В топологической динамике известны пример Бора-Жикова и лемма о разделенности Жикова.

В одной из первых его публикаций (Докл. АН СССР, 1966) ${ }^{1}$ развивается метод гармонического анализа в теории почти периодических функций, основанный на спектральном критерии почти периодичности. Этот метод позволяет детально изучить свойства ограниченных решений уравнения

$$
u^{\prime}+A u=f
$$

с постоянным (операторным) коэффициентом. В этой же работе получено усиление одного результата Ч. Фояша и С. Д. Зайдмана о почти периодичности компактных решений таких уравнений, установленного ими с помощью известной теоремы о представлении вполне неунитарной части сжатия в гильбертовом пространстве. Известная теорема Эллиса, утверждающая, что обволакивающая полугруппа дистальной динамической системы есть группа, применяется в работе В.В. Жикова "Об одной задаче Бохнера и Неймана” (Матем. заметки, 1968) для получения следующего условия почти периодичности компактных решений дифференциальных уравнений с постоянными коэффициентами: если уравнение $v^{\prime}=A^{*} v$ разрешимо на всей оси для всюду плотного множества начальных условий и решения ограничены, то каждое компактное решение уравнения $u^{\prime}=A u$ является почти периодическим. Отсюда следует классический результат С. Бохнера и Дж. фон Неймана о почти периодичности компактного решения линейного дифференциального уравнения порядка $n$ с нормальными и коммутирующими операторными коэффициентами.

Важный цикл работ В. В. Жикова посвящен разрешимости в классах почти периодических функций неавтономных линейных дифференциальных уравнений с почти периодическими коэффициентами, а также нелинейных уравнений. Первой в этом цикле стоит большая статья "K проблеме существования почти-периодических решений дифференциальных и операторных уравнений", опубликованная в научных трудах Владимирского политехнического института в 1969 г. С помощью дистальных расширений в ней передоказана достаточность условия почти периодичности решений полиномиальных уравнений с почти периодическими коэффициентами: оно заключается в отделенности от нуля соответствующего дискриминанта. Это условие было получено А. О. Вальтером и несколько позднее Х. Бором и Д. А. Фландерсом. Подход, использующий свойство дистальности, привел В.В. Жикова к построению такой почти периодической функции $f$ без нулей, что решения уравнения $z^{2}=f(t)$ не являются даже почти периодическими по Левитану. Значительная часть упомянутой статьи посвящена изучению вопроса о существовании тех или иных классов почти периодических решений дифференциальных уравнений $u_{t}^{\prime}=f(u ; t)$ с правой частью, почти периодически зависящей от $t$. Найдены весьма общие условия, при которых

\footnotetext{
13десь и далее, ссылаясь на публикации, мы указываем только название журнала и год выхода работы. Этой информации достаточно для поиска в указанных выше базах.
} 
существуют почти периодические решения таких уравнений по Левитану (и Безиковичу), в то время как почти периодических решений по Бору может не быть.

Существенный вклад внес В. В. Жиков в теорию Фавара разрешимости векторных уравнений вида

$$
u^{\prime}=A(t) u+f(t)
$$

с почти периодическими оператор-функцией $A(t)$ и вектор-функцией $f(t)$. Теория Фавара дает условия существования почти периодических решений таких уравнений в конечномерных пространствах. Л. Америо и Г. Прузе перенесли метод Фавара на равномерно выпуклые банаховы пространства, но получили при этом лишь условия для существования слабых почти периодических решений. Василий Васильевич предложил совершенно новый "негеометрический” метод, который дает условия существования слабых почти периодических решений не только в равномерно выпуклых, но и в произвольных банаховых пространствах. При этом сформулированные им некоторые усиления этих условий гарантируют компактность слабых почти периодических решений, что обеспечивает их обычную почти периодичность.

Еще в заметке 1970 г. в "Докладах АН СССР" В. В. Жиков анонсировал результаты о существовании почти периодических решений нелинейных дифференциальных уравнений, а именно параболического уравнения вида

$$
u^{\prime}+A(t) u=0
$$

и гиперболического уравнения вида

$$
u^{\prime \prime}+\varphi(u)+Q x=f,
$$

в предположении свойств монотонности:

(а) для всяких двух решений $u_{1}, u_{2}$ функция $\left\|u_{1}-u_{2}\right\|$ возрастает по $t$ в первом случае и

(b) оператор $\varphi$ является монотонным - во втором.

Подробному изложению теории Фавара, дополненному результатами о разрешимости нелинейных уравнений в классе почти периодических функций, посвящен обзор В. В. Жикова и Б. М. Левитана (Успехи матем. наук, 1977) и совместная с Б. М. Левитаном монография "Почти-периодические функции и дифференциальные уравнения" (Изд-во Моск. ун-та, 1978). Этот обзор и монография относятся к числу наиболее цитируемых публикаций В. В. Жикова.

2. В пятидесятые и шестидесятые годы прошлого века интенсивно развивалась тематика, связанная с обратной задачей Штурма-Лиувилля и одномерным оператором Шрёдингера. Одной из важнейшей в этой тематике явилась работа И. М. Гельфанда и Б. М. Левитана о восстановлении потенциала оператора по его спектральной функции (в случае дискретного спектра - по собственным значениям и нормировочным числам). Методы доказательства в этой работе существенно использовали предположения о гладкости потенциала. В известной работе "Об обратных задачах Штурма-Лиувилля на конечном отрезке" (Изв. АН СССР, 1967) Василию Васильевичу удалось не только избавиться от условий гладкости и найти форму уравнения Гельфанда-Левитана для произвольных суммируемых потенциалов, но и решить задачу для потенциалов, в которых фигурируют суммы дельта-функций.

3. Работами о стабилизации решений параболических уравнений и усреднении параболических дифференциальных операторов с почти периодическими коэффициентами (Матем. сб., 1977, 1979, 1982) завершился начальный этап математического творчества В.В. Жикова, связанный с изучением почти периодических функций и уравнений. В первой из этих работ в 1977 г. Василий Васильевич предложил замечательный новый метод, который дает критерий для равномерной и поточечной стабилизации решения задачи Коши для дивергентного параболического уравнения второго порядка $u_{t}=A(x, t) u$ без младших коэффициентов, с ограниченной начальной функцией. Этот метод сразу стал общепринятым и получил название "метод Жикова". Ранее такого рода теоремы доказывались в основном для уравнений с постоянными коэффициентами (например, для уравнения теплопроводности) и базировались 
на применении тонких средств типа тауберовых теорем Н. Винера. Метод Жикова, основанный на применении метода усреднений в соединении с методами функционального анализа, дает критерий стабилизации решений в терминах явно предъявляемого так называемого $\lambda$-функционала. В случае оператора Лапласа в правой части функционал $\lambda$ является взвешенным шаровым средним, а для оператора $A(x, t)$ с матричными коэффициентами $\left\{a_{i j}(x, t)\right\}$, удовлетворяющими при $t \rightarrow \infty$ предельным условиям Гущина-Михайлова, функционал $\lambda$ является взвешенным средним по эллипсоидам, соответствующим приведенной к нормальной форме матрице, обратной к предельной.

Этот результат В. В. Жикова послужил отправной точкой, определившей характер исследований по стабилизации решений задачи Коши для многих других математиков. Сам Василий Васильевич продолжил развитие этой тематики и в 1983 г. предложил метод, который гарантирует стабилизацию решения задачи Коши для широкого класса близких уравнений при условии, что стабилизация имеет место для простейшего представителя данного класса. При выполнении условия среднеквадратичной малости отклонения от нуля разности матриц диффузии необходимое и достаточное условие близости двух решений состоит в том, чтобы функционал шарового среднего был равен нулю для разности начальных функций. Из этого получается эффективный критерий поточечной стабилизации. Критерий равномерной стабилизации В. В. Жиковым также был получен.

В 1989 г. Василий Васильевич развил еще один новый и неожиданный метод исследования стабилизации решений параболических уравнений, основанный на применении теорем типа центральной предельной теоремы и оценок Берри-Эссеена. Ранее эти оценки играли большую роль в предельных теоремах теории вероятностей. Теперь метод Жикова находит широкое применение в вопросах стабилизации решений. В последних работах В.В. Жиков применил свой метод для установления необходимых и достаточных условий равномерной стабилизации решения задачи Коши для нелинейного параболического уравнения с $p$-лапласианом в главной части.

4. Прикладные задачи вычислительной математики и внутренняя логика развития теории дифференциальных уравнений с частными производными привели в 70-е годы прошлого века к активному развитию техники усреднения дифференциальных операторов, и эта тема стала одной из основных, над которыми работал В. В. Жиков. В сотрудничестве с С. М. Козловым и О.А. Олейник им был получен ряд фундаментальных результатов в теории усреднения и $G$-сходимости эллиптических и параболических операторов. В достаточно общей ситуации были введены существенные для разрабатываемого формализма функции, получившие в дальнейшем название корректоров. Для операторов с периодическими коэффициентами такие функции были введены Н. С. Бахваловым. Однако в работах В. В. Жикова, С. М. Козлова и О.А. Олейник корректоры определялись для существенно более широких классов операторов и стали важным инструментом при исследовании различных свойств $G$-сходимости. Отметим, что в этих работах впервые было дано систематическое и полное описание основных свойств такой сходимости для общих параболических и эллиптических дифференциальных операторов в дивергентной форме.

В это же время в связи с различными моделями в теории пористых сред, биологическими и популяционными моделями возник большой интерес к усреднению операторов, имеющих непериодическую структуру. Василий Васильевич в совместных работах с С. М. Козловым и О.А. Олейник получил ряд результатов об усреднении эллиптических и параболических уравнений и систем с почти периодическими и случайными коэффициентами, в том числе для задач теории упругости.

Еще одна область, где Василием Васильевичем были получены очень важные результаты, - это усреднение операторов с младшими членами и сингулярно возмущенных параболических операторов. Одна из основных проблем здесь - доказательство равномерных априорных оценок. Идея В. В. Жикова заключалась в том, чтобы 
усреднять такие уравнения в пространствах со специально подобранным весом, причем (как в периодическом, так и в квазипериодическом случае) вес выбирается как решение некоторой вспомогательной задачи на периоде. При таком подходе удается получить равномерные по параметру априорные оценки. Позже развитие этих идей привело к возникновению техники усреднения в движущихся координатах.

Занимаясь задачами в перфорированных областях, В. В. Жиков приходит к созданию теории эллиптических уравнений и операторов, заданных на тонких и сингулярных структурах, а также на пространствах $\mathbb{R}^{n}$ с борелевскими мерами. Были введены соответствующие соболевские пространства, определены решения эллиптических дифференциальных уравнений и построены отвечающие им операторы. Затем исследовались макроскопические свойства таких структур. Эта теория позволила создать единый подход для изучения задач усреднения в областях с негладкой микроструктурой, в областях асимптотически малого объема, для эллиптических операторов с вырождающимися и неограниченными коэффициентами.

Для усреднения мер и тонких областей с периодической микроструктурой В. В. Жиков адаптировал метод двухмасштабной сходимости. Техника двухмасштабной сходимости оказалась очень удобным инструментом при усреднении сингулярных структур. Позднее В.В. Жиков совместно с А.Л. Пятницким распространил метод двухмасштабной сходимости на случайные статистически однородные сингулярные структуры и меры.

Важное место в творческом наследии Василия Васильевича занимают задачи усреднения системы теории упругости, заданной на тонких периодических структурах. Существенная часть исследований в этой области выполнялась совместно с С. Е. Пастуховой. Выяснилось, что, в отличие от скалярных операторов, предельное поведение решений задачи теории упругости существенно зависит от соотношения скоростей убывания размера периода и толщины структуры. Изучалось также поведение константы в неравенстве Корна для тонких структур. Это неравенство служит одним из ключевых инструментов при исследовании системы теории упругости.

Высокую оценку специалистов получил красивый результат В. В. Жикова по асимптотике спектра в задаче двойной пористости. Эта задача возникает при описании процессов в $\varepsilon$-периодических средах с высококонтрастными микроструктурами. Соответствующие дифференциальные операторы асимптотически вырождаются на части микроструктуры. Изучая эту задачу, В.В.Жиков описал спектр предельного двухмасштабного оператора и доказал сходимость спектров исходных операторов к спектру предельного. Другие спектральные задачи для эллиптических операторов в высококонтрастных и сильно анизотропных средах изучались В.В. ЖКиовым в совместных проектах с В.П. Смышляевым и К. Д. Чередниченко, а для задач теории упругости - с С. Е. Пастуховой.

В.В. Жиков существенно расширил теорию двухмасштабной сходимости и ввел фундаментальное понятие сильной двухмасштабной резольвентной сходимости. На этой основе он предложил свой метод точного нахождения лакун в спектре некоторых операторов с периодическими коэффициентами, имеющих важные приложения в теории фотонных кристаллов. Метод Жикова сразу оказался востребованным и получил развитие в работах многих отечественных и зарубежных математиков. Работы В.В. Жикова "Об одном расширении и применении метода двухмасштабной сходимости" (Матем. сб., 2000) и "О лакунах в спектре некоторых дивергентных эллиптических операторов с периодическими коэффициентами” (Алгебра и анализ, 2004) оказались прорывными и по сей день широко цитируются специалистами.

5. В 1980-х годах внимание многих математиков, в том числе и В.В. Жикова, привлекла следующая проблема. При усреднении задач в перфорированных областях требовалась существенная гладкость перфорации. Интуитивно было ясно, что требование гладкости завышено. Однако используемые методы опирались на технику 
продолжения решений внутрь областей перфорации с сохранением оценок на производные, применение которых возможно лишь при определенной гладкости границ перфорации.

Шаг за шагом В.В. Жиков идет к тому, чтобы избавиться от условий гладкости. Сначала требование гладкости удается ослабить за счет построения продолжения в пространствах с более слабыми нормами. Однако это не решило проблему полностью. Василий Васильевич продолжил работать над этой задачей: он ввел сначала понятие 2-связной, а затем и $p$-связной области и показал, что критерием наличия эффективного оператора служит только условие связности. Такой подход позволил полностью отказаться от операторов продолжения, а в случае перфорированных областей дал возможность получить результаты об усреднении без каких-либо предположений о гладкости.

Понятие связности оказалось полезным и в решении других задач. С его помощью В.В. Жикову удалось решить проблему Хаммерсли-Кестена (J. Hammersley, H. Kesten) в теории протекания и проблему диффузии в областях типа внешности плотной кубической упаковки шаров. На этом же пути Василий Васильевич дал математическое объяснение феномена фрактальной проводимости и диффузии через фрактальную щель, чему была посвящена его известная работа "Связность и усреднение. Примеры фрактальной проводимости" (Матем. сб., 1996). В настоящее время техника Жикова широко применяется в задачах усреднения и именуется Zhikov measure approach.

6. В 1982 г. В. В. Жиков начинает свои знаменитые исследования по нестандартным вариационным задачам и так называемому эффекту Лаврентьева. Эффект заключается в том, что с одним и тем же функционалом можно связать по крайней мере две различные задачи Дирихле. В задачах одного типа минимизация проходит по всем функциям из естественной области определения функционала, а в задачах другого типа - только по классу гладких функций. Эффект открыл М. А. Лаврентьев при изучении одномерной вариационной задачи для функционала

$$
\int_{0}^{1} f\left(x, u(x), u^{\prime}(x)\right) d x
$$

отвечая на вопрос, поставленный в 1922 г. Л. Тонелли. В серии работ В. В. ЖКиков обращается к существенно более сложным многомерным вариационным задачам. Итогом этих работ стала теория вариационных задач с интегрантами нестандартного роста, например вида $|\xi|^{p(x)}$. В ее построении существенную роль сыграли его предшествующие исследования по задачам усреднения. Василий Васильевич построил примеры и контрпримеры к эффекту Лаврентьева, внес общепризнанный вклад в теорию соболевских пространств с переменным показателем $p(x)$, доказал разрешимость и обнаружил новые свойства решений нелинейных эллиптических и параболических уравнений с нестандартными условиями коэрцитивности и роста, построил теорию усреднения и Г-сходимости в присутствии эффекта Лаврентьева. Перечень достижений В. В. ЖКикова за 35 лет работы в этой тематике можно продолжать. Но здесь особо отметим лишь один результат - об условиях плотности гладких функций в пространстве Соболева с переменным показателем $p(x)$. Сначала в 1995 г. В. В. Жиков и его китайский коллега Fan Xiangling (который проходил стажировку в России) совместно нашли достаточное для плотности логарифмическое условие на показатель $p(x)$ (Fan-Zhikov condition), а в 2004 г. ими было найдено аналогичное условие на $p(x)$ с двойным логарифмом.

Работы В.В. Жикова, связанные с эффектом Лаврентьева, широко цитируются и признаны прорывными - в частности, его статья "On Lavrentiev's phenomenon" (Russ. J. Math. Phys., 1995) цитируется уже в 158 работах в базе MathSciNet, причем это число продолжает расти.

7. В начале 2000-х В. В. Жиков получил фундаментальные результаты по усреднению задач теории упругости на сингулярных и тонких структурах в объемной работе 
“Усреднение задач теории упругости на сингулярных структурах" (Изв. РАН, 2002), а также продолжил свои замечательные исследования по эффекту Лаврентьева в статье "K проблеме предельного перехода в дивергентных неравномерно эллиптических уравнениях" (Функц. анализ и его прил., 2001). К числу его лучших работ следует отнести заметку "Об операторных оценках в теории усреднения" (Докл. РАН, 2005). В ней рассматривалась операторная оценка погрешности усреднения для эллиптического дифференциального оператора с $\varepsilon$-периодическими коэффициентами и была доказана оценка сходимости порядка $O(\varepsilon)$ при $\varepsilon \rightarrow 0$ в операторной $L^{2}$-норме резольвенты исходного оператора к резольвенте предельного оператора.

Подобного рода вопросы, связанные с операторными оценками усреднения для полугрупп, порождаемых параболическими уравнениями, в операторных $L^{\infty}$-нормах поднимались и решались В.В. Жиковым ранее в его работе "Спектральный подход к асимптотическим задачам диффузии" (Дифференц. уравнения, 1989). Само название статьи указывает на метод, который был применен для доказательства оценок усреднения, а также других асимптотических результатов типа центральной предельной теоремы или оценок равносходимости при стабилизации. После публикации в 2003 г. обширных исследований М. Ш. Бирмана и Т. А. Суслиной по операторным оценкам усреднения (в них также использовался берущий свое начало в работах И. М. Гельфанда спектральный метод, основанный на разложении Флоке-Блоха) В. В. Жиков вновь вернулся к старой для себя тематике. Из "спортивного" интереса Василий Васильевич задался целью найти подход к операторным оценкам, близкий к классическому методу асимптотических разложений, что и было исполнено в упомянутой выше короткой заметке в "Докладах РАН" (2005). Появился новый метод получения операторных оценок усреднения, чрезвычайно прозрачный, концептуально простой и универсальный по сферам приложения. В его основе лежит идея введения дополнительного параметра интегрирования через сдвиг в коэффициентах оператора. Этот прием снимает многие проблемы, возникающие в оценках в тех случаях, когда минимальны требования на данные задачи (на коэффициенты оператора, правую часть уравнения, начальное данное в задаче Коши).

Метод сдвига получил широкое применение, а сама заметка приобрела необычно высокую для кратких сообщений цитируемость. Этот метод был развит в серии совместных с С. Е. Пастуховой работ. В них рассматривались самые различные уравнения: эллиптические и параболические, скалярные и векторные, вырожденные и высокого порядка, притом не обязательно с периодическими коэффициентами, например, коэффициенты могли быть многомасштабными или квазипериодическими. Сам метод сдвига трансформировался в некоторых задачах в свою модификацию - метод сглаживания по Стеклову. Итогом многолетней деятельности по операторным оценкам усреднения стал совместный с С. Е. Пастуховой обзор, опубликованный в 2016 г. в журнале "Успехи математических наук".

8. В последнее десятилетие В.В. Жиков обратился к задачам теории нелинейных уравнений с частными производными и добился выдающихся результатов. Ему удалось решить задачу о термисторе (термистор - полупроводниковый прибор для измерения температуры и потенциала электрического поля).

Одна из самых трудных проблем, решенных В. В. Жиковым, касается разрешимости обобщенных уравнений Навье-Стокса с показателем нелинейности $p \geqslant p_{0}$ в вязком члене. Нижняя граница $p_{0}$ допустимых показателей нелинейности в вязком члене была сдвинута в трехмерном случае до значения $(3+\sqrt{39}) / 5$, меньшего двух, чего не удавалось сделать долгое время после работ О. А. Ладыженской и Ж.-Л. Лионса 1960-х годов, когда этот вопрос был поднят.

В. В. Жиков разработал технику перехода к пределу в нелинейных задачах, что позволило ему решить трудную проблему "пространственного" усреднения уравнений Навье-Стокса для электрореологической жидкости, в которых показатель нелинейности в вязком члене переменный; более того, этот показатель может представлять 
собой $\varepsilon$-периодическую функцию пространственного переменного, быстро осциллирующую при $\varepsilon \rightarrow 0$.

9. Одним из последних выдающихся достижений Василия Васильевича, полученных им уже после 70-летия, стало доказательство известной гипотезы Де Джорджи о достаточных условиях плотности гладких функций в весовом пространстве Соболева $W^{2,1}(\varrho)$ с весом $\varrho \geqslant 0$ на $\mathbb{R}^{n}$. Этот класс по определению состоит из функций $f$, локально принадлежащих обычному (без веса) соболевскому пространству $W^{1,1}$, для которых интегрируемы $|f|^{2} \varrho$ и $|\nabla f|^{2} \varrho$. Гипотеза, остававшаяся открытой десятилетия и не поддававшаяся усилиям многих известных исследователей, состояла в том, что достаточным условием для плотности класса гладких финитных функций в классе $W^{2,1}(\varrho)$ является локальная интегрируемость функций $\exp (t \varrho), \exp \left(t \varrho^{-1}\right)$ при всех $t>0$. Были известны примеры, показывающие, что локальная интегрируемость степеней $\varrho$ и $\varrho^{-1}$ не гарантирует плотность финитных функций, ряд ярких примеров такого рода был построен самим Жиковым. Как это нередко бывает с трудными задачами, для положительного решения проблемы Де Джорджи пришлось доказывать более сильное утверждение. Оказалось, что достаточным является следующее, более слабое и неожиданное условие (условие Жикова):

$$
\sup _{n} n^{-2}\left(\int_{U} \varrho^{n} d x\right)^{1 / n}\left(\int_{U} \varrho^{-n} d x\right)^{1 / n}<\infty
$$

для всякого шара $U \subset \mathbb{R}^{d}$. Для доказательства Василий Васильевич изучал вырожденное эллиптическое уравнение вида

$$
-\operatorname{div}(\varrho A \nabla v)+v \varrho=f \varrho,
$$

где $A$ - эрмитова матрица, подчиненная условию $\lambda^{-1} \leqslant A \leqslant \lambda$, и $f \in C_{0}^{\infty}\left(\mathbb{R}^{n}\right)$. Чтобы проверить, что замыкание множества финитных функций совпадает со всем классом Соболева $W^{2,1}(\varrho)$, надо уметь доказывать отсутствие ненулевых решений уравнения

$$
\operatorname{div}(\varrho \nabla u)=0
$$

без младших членов в обычном классе Соболева $W_{0}^{1,1}(B)$ с нулевым граничным условием и интегрируемой производной лишь первого порядка, что, как известно, заметно усложняет исследование единственности. Успех в доказательстве гипотезы Де Джорджи был достигнут Василием Васильевичем именно за счет значительного продвижения в этой представляющей большой самостоятельный интерес задаче о единственности решений вырожденного эллиптического уравнения. После публикации решения стало понятно, почему это выдающееся достижение оказалось под силу именно Жикову: ведь в целом ряде его предшествующих работ по эффекту Лаврентьева, весовым классам Соболева, вырожденным уравнениям звучали очень близкие мотивы. В последние годы В.В. Жиков работал над этой тематикой совместно с М. Д. Сурначевым.

Василий Васильевич основал замечательную школу. Среди его учеников 16 кандидатов и 7 докторов наук.

Василий Васильевич Жиков был не только выдающимся ученым и уникальным руководителем, но и чутким, добрым, благородным и открытым человеком, всегда готовым помочь. Благодарная память о нем навсегда сохранится в сердцах его коллег, учеников и всех соприкасавшихся с ним.

Ю.А. Алхутов, И. В. Асташова, В. И. Богачев, В.Н. Денисов, В.В. Козлов, С.Е. Пастухова, А.Л. Пятницкий, B. А. Садовничий, А. М. Степин, А.С. Шамаев, А. А. Шкаликов 